\title{
Anti-inflammatory activity of cinnamon water extract in vivo and in vitro LPS-induced models
}

\author{
Joung-Woo Hong', Ga-Eun Yang ${ }^{1}$, Yoon Bum Kim², Seok Hyun Eom³ ${ }^{3}$ Jae-Hwan Lew ${ }^{1}$ and Hee Kang ${ }^{1 *}$
}

\begin{abstract}
Background: Cinnamon bark is one of the most popular herbal ingredients in traditional oriental medicine and possesses diverse pharmacological activities including anti-bacterial, anti-viral, and anti-cancer properties. The goal of this study is to investigate the in vivo and in vitro inhibitory effect of cinnamon water extract (CWE) on lipopolysaccharide (LPS)-induced tumor necrosis factor (TNF)-a and its underlying intracellular mechanisms.

Methods: CWE was orally administrated to mice for 6 days prior to intraperitoneal injection of LPS. Serum levels of TNF-a and interleukin (IL)- 6 were determined 1 hour after LPS stimulation. Peritoneal macrophages from thioglycollate-injected mice were isolated and assayed for viability, cytokine expression and signaling molecules upon LPS stimulation. CWE was further fractioned according to molecular size, and the levels of total polyphenols and biological activities of each fraction were measured.

Results: The oral administration of CWE to mice significantly decreased the serum levels of TNF-a and IL-6. CWE treatment in vitro decreased the mRNA expression of TNF-a. CWE blocked the LPS-induced degradation of IKBa as well as the activation of JNK, P38 and ERK1/2. Furthermore, size-based fractionation of CWE showed that the observed inhibitory effect of CWE in vitro occurred in the fraction containing the highest level of total polyphenols.

Conclusions: Treatment with CWE decreased LPS-induced TNF-a in serum. In vitro inhibition of TNF-a gene by CWE may occur via the modulation of IKBa degradation and JNK, p38, and ERK1/2 activation. Our results also indicate that the observed anti-inflammatory action of CWE may originate from the presence of polyphenols.
\end{abstract}

Keywords: Cinnamon, Anti-inflammatory activity, TNF-a, Signaling, Polyphenols

\section{Background}

The bark of cinnamon has been used not only as a spice and tea, but also as one of the key components of herbal remedies for the common cold, cardiovascular disease, and chronic gastrointestinal and gynecological disorders in oriental herbal medicine. Accordingly, extensive studies on the pharmacological activities of the cinnamon bark have been conducted, indicating that cinnamon bark is involved in a vast range of pathological and physiological events. For instance, essential oil and water-based extracts from cinnamon have been shown to be effective against pathogenic microbes, viruses, and various types of tumor cell lines [1-4]. Furthermore, it has been also reported that cinnamon bark reduces the level of serum glucose

\footnotetext{
* Correspondence: shehee@khu.ac.kr

${ }^{1}$ Graduate School of East-west Medical Science, Kyung Hee University, Yongin, Gyeonggi 446-701, South Korea

Full list of author information is available at the end of the article
}

through the enhancement of insulin-regulated glucose utilization in vivo [5,6].

Inflammation is a protective response for the purpose of removal of exogenous and endogenous harmful substances produced by injurious stimuli and is a part of the healing process in wounded tissues [7]. Since proinflammatory cytokines such as tumor necrosis factoralpha(TNF- $\alpha$ ), interleukin(IL)-1 and IL-6, lipid mediators, proteases, and oxidants produced during the typical response can cause damage to normal tissues regardless of how and where the inflammatory response is triggered, the substances involved in the inflammatory response need to be tightly regulated. If the scavenging reaction is delayed, the inflammatory response may evolve into a variety of chronic inflammatory diseases, such as atherosclerosis, rheumatoid arthritis, asthma, and neurodegenerative diseases. A vast number of molecular studies have identified several target molecules involved in inflammatory 
changes, and most anti-inflammatory drugs currently used suppress the biosynthesis of the inflammatory mediators mentioned earlier [8].

Previous studies have indicated that the major pharmacological activities of cinnamon bark, such as its anti-bacterial, anti-inflammatory, anti-viral, and anticancer effects are derived from essential oils such as cinnamaldehyde [1,9-11]. However, since cinnamon bark has been typically used as in the form of a water extract, where the volatile ingredients are seldom found, it is likely that the established pharmacological activities of cinnamon bark depend on a mixture comprised of a variety of water-soluble components, thereby ensuring its safety as a traditional remedy. Recently, it has been found that cinnamon bark water extract (CWE) elevates glucose uptake through the promotion of insulin sensitivity and inhibits angiogenesis through blocking vascular endothelial growth factor 2 signaling $[12,13]$. These results indicate that the observed pharmacological activities may have originated from polyphenolic compounds in CWE.

In this study, we investigated the in vivo and in vitro effects of CWE on lipopolysaccharide (LPS)-induced TNF- $\alpha$ and its underlying intracellular mechanisms. We also fractioned CWE according to molecular size to determine whether there exists a positive correlation between the anti-inflammatory activity of CWE and the amount of polyphenolic compounds.

\section{Methods}

\section{Preparation of cinnamon water extract}

Cinnamon bark (Cinnamomi cassia $\mathrm{P}_{\mathrm{RESL}}$ ) of Vietnamese origin was purchased from Omni Herb (Daegu, South Korea). The plant was identified by Professor Choi of the Department of Herbology at Kyung Hee University. A voucher specimen sample (CC-2011) was deposited at the Laboratory of Herbology at Kyung Hee University. The plant was pulverized and soaked in one volume of water for 48 hours at room temperature, and further dissolved by sonication for 1 hour. The extract was filtered and evaporated using a freeze dryer (EYELA, Japan) at $-70^{\circ} \mathrm{C}$. The yield of CWE was about $3.62 \%$. For size fractionation, $1.28 \mathrm{~g}$ of CWE was dissolved in $30 \mathrm{ml}$ of distilled water and fractions were collected using $3 \mathrm{kDa}$ and $10 \mathrm{kDa}$ Amicon Ultra Centrifugal Filter device

(Millipore, Ireland). The yields of a low molecular weight (MW) fraction (below $3 \mathrm{kDa}$ ), a middle MW fraction (between $3 \mathrm{kDa}$ and $10 \mathrm{kDa}$ ), and a high MW fraction (over $10 \mathrm{kDa}$ ) were $60 \%, 15 \%$ and $25 \%$ of CWE, respectively. All the final samples were dissolved in PBS and sterilized by passing through a $0.22-\mu \mathrm{m}$ syringe filter.

\section{Animals}

Eight-week-old male BALB/c mice were purchased from the Korean branch of Taconic, SamTaco (Osan, Korea) and fed rodent chow and water ad libitum in a temperature- and humidity-controlled pathogen-free animal facility at the Medical Center of Kyung Hee University Hospital. Mice were maintained in accordance with the Guide for the Care and Use of Laboratory Animals issued by the US National Research Council (1996), and the protocol KHMC-IACUC12-006 was approved by the Kyung Hee University Medical Center Institutional Animal Care and Use committee.

\section{In vivo LPS injection}

CWE (20, 100 or $500 \mathrm{mg} / \mathrm{kg}$ of body weight) was given to mice via oral gavage for 6 days. Control mice received an equal volume of normal saline during the experimental period. Each group consisted of 12 mice. On day 7 , LPS (serotype 055:B5; Sigma, St. Louis, MO, USA) $(1.3 \mathrm{mg} / \mathrm{kg})$ was injected intraperitoneally 1 hour before blood sampling. Blood was obtained by cardiac puncture. As a reference drug, dexamethasone (Sigma) $(5 \mathrm{mg} / \mathrm{kg}$ ) was injected intraperitoneally 18 hours before the LPS injection. Blood samples were centrifuged at $800 \mathrm{~g}$ for $20 \mathrm{~min}$. The serum samples obtained were stored at $-20^{\circ} \mathrm{C}$ until used.

\section{Isolation and culture of peritoneal macrophages}

For the use of in vitro culture of macrophages, normal mice were injected intraperitoneally with $2 \mathrm{ml}$ of sterile thioglycollate medium (BD, France), and macrophages were collected three days later by peritoneal gavage with cold Dulbecco's modified Eagle's medium (DMEM). The recovered peritoneal fluid was washed by centrifugation. The cells were resuspended in DMEM with 10\% fetal bovine serum and incubated for 3 hours at $37^{\circ} \mathrm{C}$ with $5 \%$ $\mathrm{CO}_{2}$. Non-adherent cells were removed.

\section{Viability assay}

Cell viability was determined using the MTT method. Macrophages were seeded in 96-well plates and treated with 10, 50,100, 200, and $400 \mu \mathrm{g} / \mathrm{ml} \mathrm{CWE} \mathrm{in} \mathrm{the} \mathrm{presence}$ or absence of LPS for 24 hours. Ten microliters of MTT solution $(5 \mathrm{mg} / \mathrm{ml}$ ) (Sigma) was added to each well and, after 2 hours of incubation, media was aspirated and 100 $\mu \mathrm{l}$ of dimethyl sulfoxide (DMSO) (Sigma) was added. The optical density was read at $560 \mathrm{~nm}$ using a microplate reader (Molecular Devices, Sunnyvale, CA, USA).

\section{CDNA preparation and real-time PCR}

Peritoneal macrophages were seeded in 6-well plates and pre-treated with CWE for 1 hour, then stimulated with $100 \mathrm{ng} / \mathrm{ml}$ LPS for 4 hours. Total RNA was isolated using an RNeasy Mini Kit (Qiagen, Germany) and cDNA was reverse-transcribed using Superscript III reverse transcriptase (Invitrogen, Carlsbad, CA, USA). Diluted cDNA was mixed with Power SYBR Green PCR Master 
mix (Applied Biosystems, Foster City, CA, USA) and 2 pmol of primers for TNF- $\alpha$ or GAPDH and. The following forward and reverse primer sequences were used: TNF- $\alpha$, forward : $5^{\prime}$ - ATG ATC GCG GAC GTG GAA-3/ and reverse: $5^{\prime}$-AGG GCC TGG AGT TCT GGA A-3/; GAPDH, forward: 5'-GGC ATG GAC TGT GGT CAT GA-3' and reverse: $5^{\prime}$-TTC ACC ACC ATG GAG AAG GC-3'. Amplification of cDNA was performed in triplicate using a StepOne realtime PCR system (Applied Biosystems). After an initial heat denaturation at $95^{\circ} \mathrm{C}$ for $10 \mathrm{~min}$, the PCR conditions were set at $95^{\circ} \mathrm{C}$ for $15 \mathrm{~s}$ and $60^{\circ} \mathrm{C}$ for $1 \mathrm{~min}$ for 40 cycles. For each PCR, a corresponding mRNA sample without RT was included as a negative control. Quantification of each cDNA copy number was determined according to the manufacturer's protocol. The GAPDH gene was used as an endogenous control.

\section{Cytokine measurement}

The levels of cytokines from serum or cell supernatants were measured by enzyme-linked immunosorbent assay (ELISA), according to the manufacturer's protocol (BD Pharmingen, USA).

\section{Western blot analysis}

Peritoneal macrophages were seeded and pretreated with CWE for 1 hour and then stimulated with LPS for 15 min. Cells were rinsed in cold PBS and then lysed on ice in $0.1 \mathrm{ml}$ of RIPA buffer (50 mM Tris- $\mathrm{HCl}, \mathrm{pH} 7.5$; $150 \mathrm{mM} \mathrm{NaCl} ; 1 \mathrm{mM}$ EDTA; $20 \mathrm{mM} \mathrm{NaF}$; 0.5\% NP-40; and $1 \%$ Triton X-100) containing phosphatase inhibitor cocktail (Sigma) and protease inhibitor cocktail (Roche
Diagnostics, Mannheim, Germany). After centrifugation at 13,000 g for $10 \mathrm{~min}$, supernatants were collected. Protein concentrations were determined using the Bradford protein assay reagent (Bio-Rad, USA) and the samples were diluted with $6 \mathrm{x}$ sodium dodecyl sulfate(SDS) buffer and boiled for $3 \mathrm{~min}$. The samples were separated on a $10 \%$ SDS-polyacrylamide gel and were transferred to polyvinylidene fluoride membranes. The membranes were blocked with $5 \%$ skim milk in Tris-buffered saline with $0.1 \%$ Tween 20 (TBST) for 1 hour. The membranes were incubated with I $\mathrm{B} \alpha$, IKK, tubulin (Santa Cruz Biotechnology, CA, USA), phospho-IKK, phospho-IкB $\alpha$, phospho-JNK, JNK, phospho-ERK1/2, ERK1/2, phosphop38, and p38 diluted in 5\% skim milk in TBST overnight at $4{ }^{\circ} \mathrm{C}$. The blots were washed with TBST and incubated for 1 hour with anti-rabbit horseradish peroxidaseconjugated antibodies. Immunoreactive bands were visualized by chemiluminescence using ECL (GE Healthcare, Little Chalfont, Buckinghamshire, UK), according to the manufacturer's instructions.

\section{Quantitation of total polyphenols}

Total polyphenols from CWE and the size-based fractions were determined by Folin-Ciocalteau (FC) colorimetry as described previously [14]. Gallic acid solutions were used for a calibration standard curve. Twenty microliters of each fraction or total CWE in $1.58 \mathrm{ml}$ of water was incubated with $100 \mu \mathrm{l}$ of FC reagent (Sigma) for $5 \mathrm{~min}$ at room temperature. Three hundred microliters of $1.88 \mathrm{M}$ sodium carbonate solution was used to quench the FC reagent-mediated reaction to form

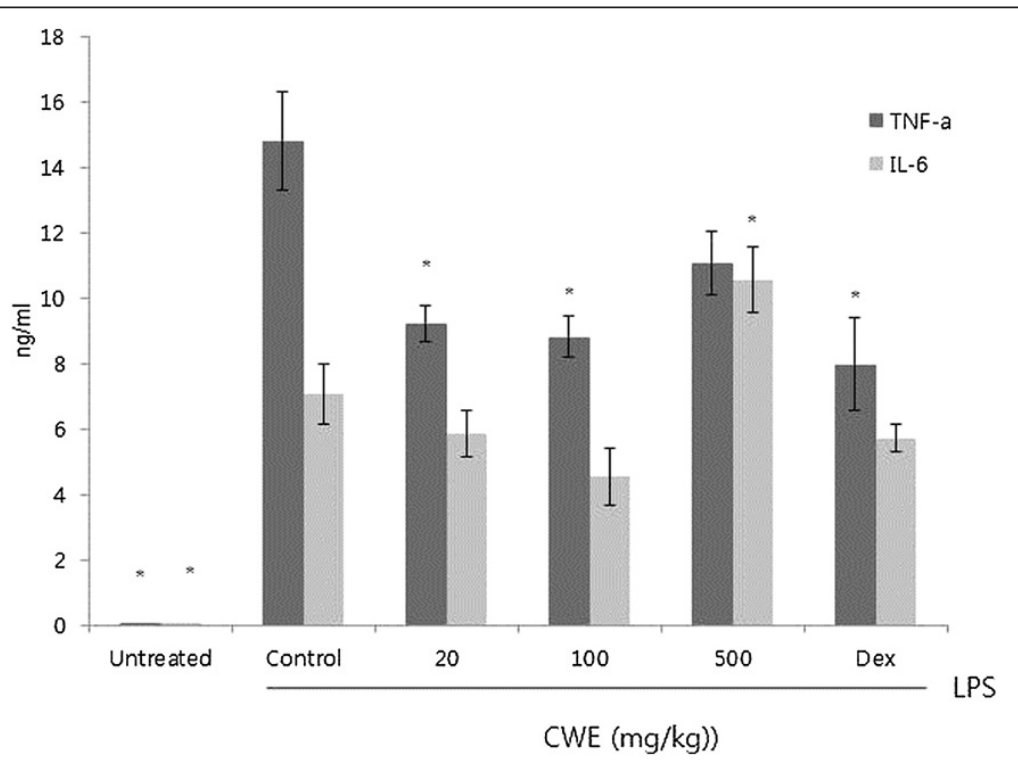

Figure 1 In vivo effect of cinnamon water extract (CWE) on levels of LPS-stimulated serum inflammatory cytokines. CWE (20, 100, and $500 \mathrm{mg} / \mathrm{kg}$ ) was orally administered to mice for 6 days before intraperitoneal injection of LPS $(1.3 \mathrm{mg} / \mathrm{kg})$. Dexamethasone (Dex) $(5 \mathrm{mg} / \mathrm{kg}$ ) was i.p. injected $18 \mathrm{~h}$ before LPS injection. Serum was obtained $1 \mathrm{~h}$ after LPS stimulation and the levels of TNF-a and IL-6 were measured by ELISA. Data are expressed as the mean \pm SEM of three independent experiments $(n=12)$. $P<0.05$ compared with the control group. 


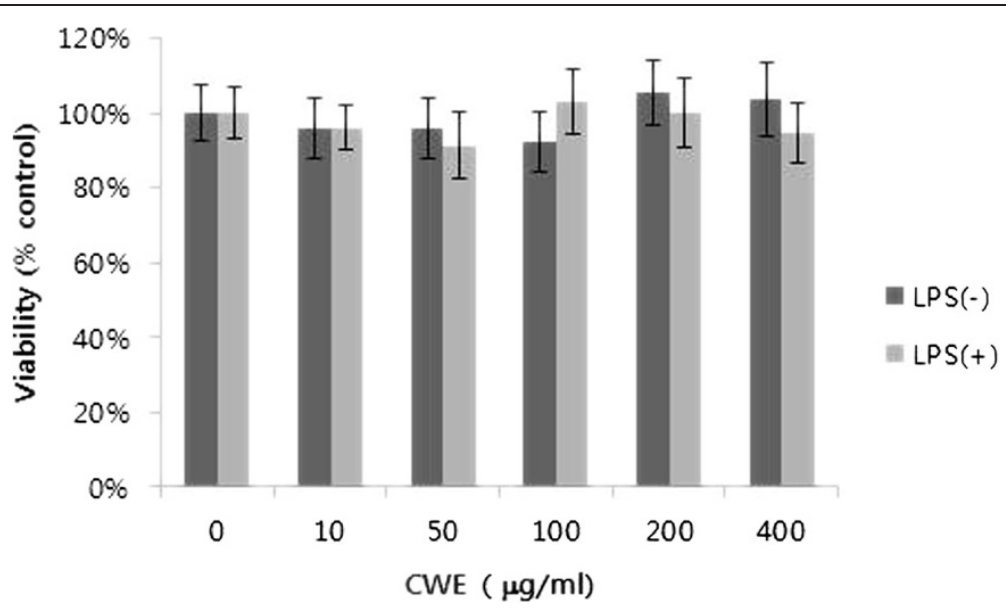

Figure 2 In vitro effect of CWE on the viability of macrophages. Peritoneal macrophages from thioglycollate-injected mice were isolated and treated with varying concentrations of CWE for 24 hours with or without LPS (100 ng/ml). Cell viability was measured by the MTT assay. Control: cells without CWE ( $0 \mu \mathrm{g} / \mathrm{ml})$. Data are expressed as the mean \pm SD of two independent experiments.
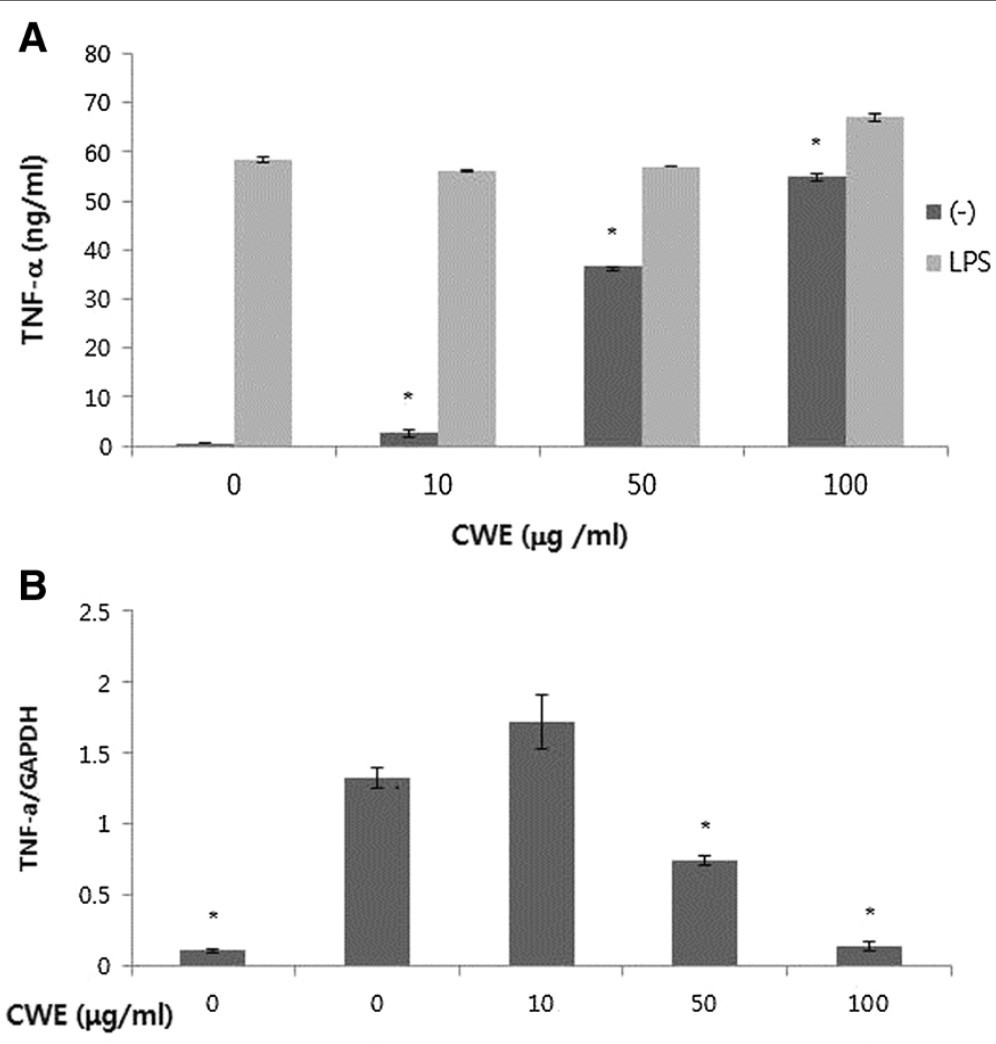

LPS

Figure 3 In vitro effect of CWE on LPS-induced TNF-a expression in macrophages. A: Peritoneal macrophages were treated with CWE for $24 \mathrm{~h}$ in the absence or presence of LPS. CWE was added $1 \mathrm{~h}$ before LPS stimulation. Supernatants were collected and cytokine levels were measured by ELISA. * $P<0.05$ compared with non-treated cells. B: Peritoneal macrophages were pretreated with CWE for $1 \mathrm{~h}$ and then stimulated with LPS for $4 \mathrm{~h}$. Total RNA was prepared and realtime RT-PCR was performed. Glyceraldehyde 3-phosphate dehydrogenase (GAPDH) was used as an internal control. * $P<0.05$ compared with cells treated with LPS only. Data are expressed as the mean \pm SD of four independent experiments. 
chromogens. After reading absorbance at $765 \mathrm{~nm}$, the concentration of polyphenols was calculated as gallic acid equivalent per gram of extract.

\section{Statistical analysis}

In vivo data are presented as mean \pm SEM. Statistical differences among the means of multiple groups were determined by using one-way ANOVA followed by the Scheffe test. In vitro data are presented as mean \pm SD. The difference between the two means was assessed using a non-paired Student's $t$-test. Calculations were carried out using SPSS version 12. P values of less than 0.05 were considered significant.

\section{Results}

In vivo effect of CWE on levels of serum tumor necrosis factor- $a$ and interleukin- 6 upon LPS stimulation

LPS is an endotoxin originating from the cell walls of gram-negative bacteria, which stimulates the expression of TNF- $\alpha$ and IL- 6 in monocytes and macrophages. These cytokines provide protective effects for the body by inducing blood clotting, leukocyte recruitment and activation of adaptive immunity, but a large quantity of such cytokines in serum results in provoking disseminated intravascular coagulation, multiple organ failure or septic shock [15]. First, we tested whether CWE treatment affects systemic inflammatory response to LPS stimulation. To this end, CWE was orally administrated to mice for 6 days before intraperitoneal injection of LPS and the release of serum TNF- $\alpha$ and IL- 6 was measured by ELISA. Based on our previous study, doses of 20,100 , and $500 \mathrm{mg} / \mathrm{kg}$ were chosen for oral administration [16]. Dexamethasone was used as a reference drug to compare the suppressive activity of CWE in the presence of LPS. Serum levels of TNF- $\alpha$ were significantly reduced with the 20 and $100 \mathrm{mg} / \mathrm{kg}$ doses, while a higher dose $(500 \mathrm{mg} / \mathrm{kg})$ showed a lesser reduction than the lower dose points and was not statistical significant (Figure 1). This pattern was observed in our previous work in which a dose-dependent reduction in serum IFN- $\gamma$ occurred only between $20-200 \mathrm{mg} / \mathrm{kg}$ of CWE [16]. In the case of IL-6, a dose-dependent decrease occurred in the 20 and $100 \mathrm{mg} / \mathrm{kg}$ dosage groups although the latter group reached statistical significance. The $500 \mathrm{mg} / \mathrm{kg}$ group showed a higher level of IL-6 than the control group. It seems that unidentified compounds beyond a critical level may interfere with the antiinflammatory components of CWE.

\section{In vitro effect of CWE on LPS-stimulated TNF-a in peritoneal macrophages}

Next, we wanted to examine the in vitro effect of CWE in macrophages, which are the major cell source of TNF- $\alpha$. We used peritoneal macrophages isolated from thioglycollate-injected mice to determine the range of CWE concentration representing no cytotoxic activity using the MTT method. Concentrations of CWE up to $400 \mu \mathrm{g} / \mathrm{ml}$ was not cytotoxic to peritoneal macrophages treated with or without LPS (Figure 2). Many herbal water extracts contain polysaccharides, which induce the secretion of TNF- $\alpha$ in non-stimulated macrophages in vitro [17]. We found that treatment with CWE alone ranging from 10 to $100 \mu \mathrm{g} / \mathrm{ml}$ stimulated the release of TNF- $\alpha$ into media in a concentration-dependent manner (Figure 3A). Such phenomenon must be due to the presence of water soluble polysaccharides in CWE. Subsequently, we examined the effects of CWE on

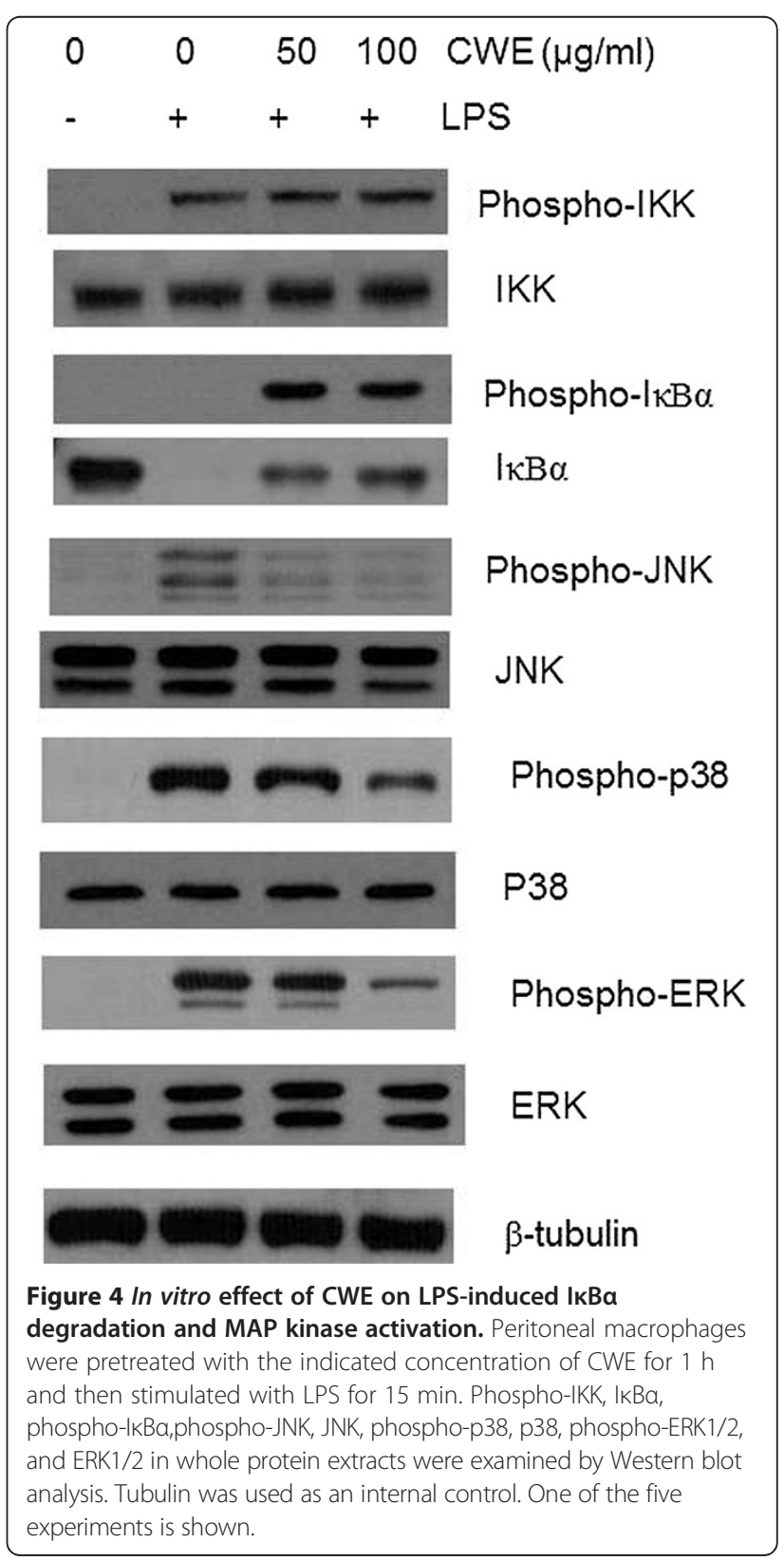


Table 1 Total polyphenols in CWE and its size-based fractions

\begin{tabular}{lllll}
\hline Polyphenols & CWE & \multicolumn{2}{l}{ CWE fractions } & \\
\cline { 3 - 5 } & & Low fraction & Middle fraction & High fraction \\
\hline & & $(<3 \mathrm{kDa})$ & $(3-10 \mathrm{kDa})$ & $(>10 \mathrm{kDa})$ \\
(mg/g extract) & $88.00 \pm 0.58$ & $19.70 \pm 0.33$ & $30.59 \pm 3.56$ & $341.18 \pm 3.33$ \\
\hline
\end{tabular}

Total polyphenolic content was measured by Folin-Ciocalteu colorimetry. Results are expressed as means $\pm S D, n=3$.

LPS-stimulated TNF- $\alpha$ secretion. There was no apparent decrease in TNF- $\alpha$ secretion by CWE (Figure 3A). However, TNF- $\alpha$ mRNA expression at $4 \mathrm{~h}$ after LPS challenge was decreased in cells treated with 50 and $100 \mu \mathrm{g} / \mathrm{ml}$ of CWE (Figure 3B). Despite its inhibition of LPS-induced TNF- $\alpha$ transcription, the unabated TNF- $\alpha$ levels in supernatant must have been caused by prior exposure of cells to CWE.

In vitro effect of CWE on activation of IKBa, JNK, p38 and ERK $1 / 2$ upon LPS stimulation

In the absence of stimuli, I $\mathrm{K} \mathrm{B} \alpha$ acts as an inhibitor to block the nuclear translocation of NFkB by masking its nuclear localization signal. Upon inflammatory stimulation, I $\mathrm{I}_{\kappa} \mathrm{B}$ is subjected to phosphorylation mediated by an upstream kinase, IKB $\alpha$ kinase (IKK), and is subsequently released from NF-kB, followed by phosphorylation-induced proteosomal degradation[18]. We tested the effect of CWE on $I_{k} B \alpha$ degradation 15 min after LPS stimulation. A time course study of $\mathrm{I} \kappa \mathrm{B} \alpha$ activity in LPS-stimulated macrophages shows that phosphorylation of $\mathrm{I} \kappa \mathrm{B} \alpha$ reaches its peak at $5 \mathrm{~min}$ after LPS stimulation and then disappears at $15 \mathrm{~min}$ while a complete degradation of IkB $\alpha$ occurs within 15 min [19]. As expected, an almost complete loss of phospho- IкB $\alpha$ as well as $I \kappa B \alpha$ was identified in macrophages treated with LPS alone (Figure 4). CWE at doses of 50 and $100 \mu \mathrm{g} / \mathrm{ml}$ inhibited I $\mathrm{KB} \alpha$ degradation, although phospho-IkB $\alpha$ was still detected. Phosphorylation of IKK was not affected. These results strongly indicate that the inhibitory effect of CWE on NF- $\mathrm{KB}$ signaling may occur downstream of the phosphorylation of $\mathrm{I} \kappa \mathrm{B} \alpha$.

Mitogen-activated protein (MAP) kinases resident in the cytoplasm of mammalian cells relay extracellular signals to the nucleus through various signal transduction pathways[20]. JNK, p38, and ERK1/2, representing the family of MAP kinases, play a critical role in LPSinduced cytokine gene expression. We tested whether CWE can inhibit the activation of JNK, p38, and ERK1/2 15 min after LPS stimulation. JNK phosphorylation was reduced at all concentrations tested and phosphorylation of p38 and ERK $1 / 2$ was inhibited at $100 \mu \mathrm{g} / \mathrm{ml}$ of CWE, implying that different constituents of CWE may act on these pathways (Figure 4). Taken together, these results show that such inhibition occurred upstream of the MAP kinases.
Inhibitory effect of the CWE fraction containing highmolecular-weight compounds on signaling molecules in LPS-stimulated macrophages

Since the effective oral dose of CWE is relatively low, it is reasonable to speculate that the nature of the active components might be small-molecule compounds. Thus we separated CWE into a low MW fraction (below $3 \mathrm{kDa}$ ), a middle MW fraction (between $3 \mathrm{kDa}$ and $10 \mathrm{kDa}$ ), and a high MW fraction (over $10 \mathrm{kDa}$ ). Since the insulin-like and antioxidant activities of CWE originate from polyphenolic compounds, we wanted to determine the polyphenol content in these fractions [12]. As shown in Table 1, the highest concentration of total

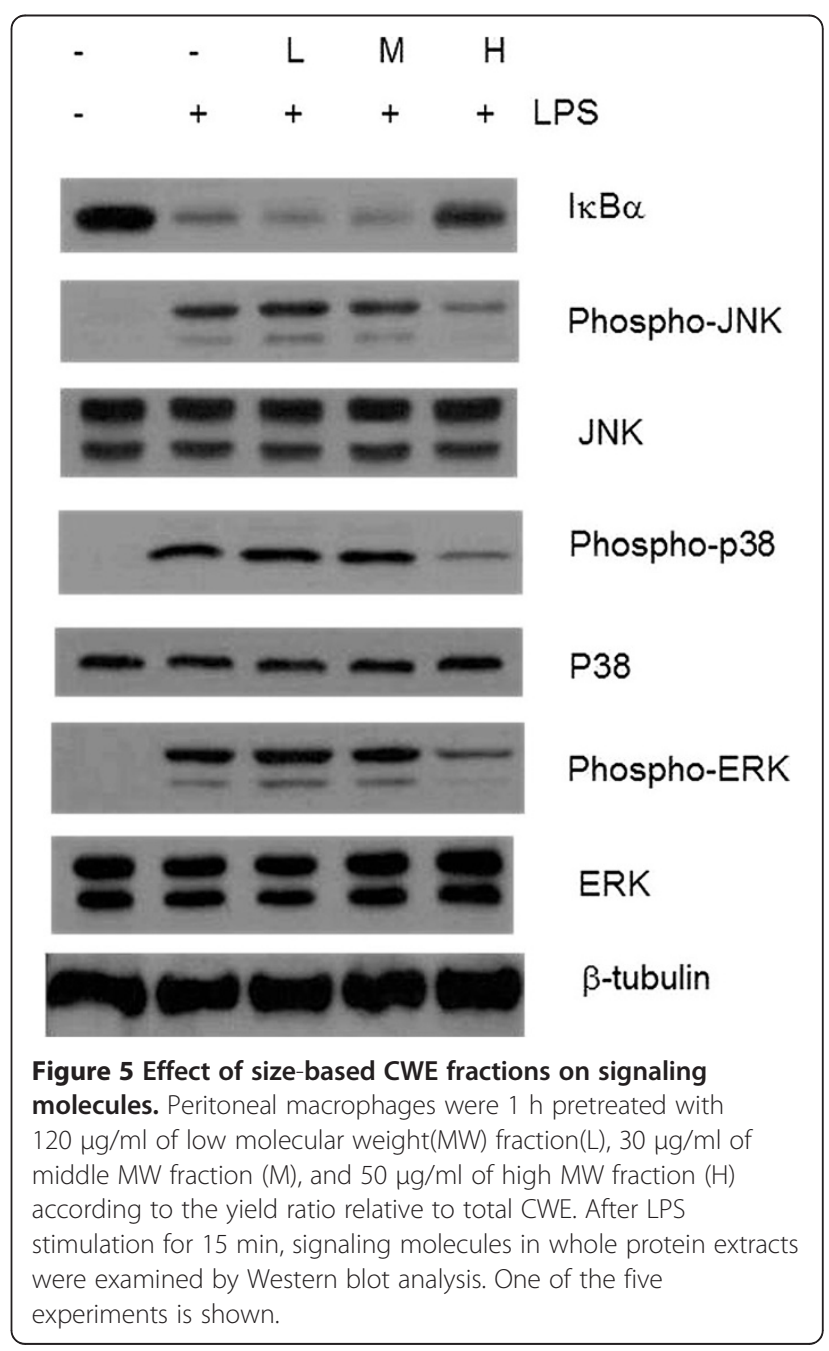


polyphenols was observed in the high MW fraction, being more than ten-fold than that of the low or middle MW fraction. Next, based on the fraction yield ratio relative to the total CWE, $120 \mu \mathrm{g} / \mathrm{ml}, 30 \mu \mathrm{g} / \mathrm{ml}$ and $50 \mu \mathrm{g} / \mathrm{ml}$ of the low, middle and high MW fractions were added to macrophages and their effects on signaling molecules were examined. Suppression of the degradation of IKB $\alpha$ and phosphorylation of JNK, p38 and ERK $1 / 2$ was detected only in cells treated with the high MW fraction (Figure 5). These findings indicate that the inhibitory activity of CWE in vitro is derived from the polyphenol-rich fraction.

\section{Discussion}

In this study, we present in vivo and in vitro evidence that CWE inhibits expression of TNF- $\alpha$. In addition, LPS-induced ІкB $\alpha$ degradation and MAP kinase phosphorylation in macrophages was strongly inhibited by the polyphenol-rich CWE fraction.

Macrophages are phagocytic cells that play a critical role in clearing foreign materials, invading bacteria and cellular debris produced by tissue injuries [21]. Phagocytes such as macrophages contain a variety of pattern-recognition receptors (PRRs), which specifically recognize foreign organisms and modified self ligand. Toll-like receptors (TLRs), complement receptor 3 and scavenger receptors are affiliated members of the PRR family. Among them, LPS uses TLR-mediated signaling pathways such as NF$\kappa B$ and MAP kinases to stimulate TNF- $\alpha$ and IL- 6 in macrophages. Oral administration of CWE decreased serum levels of LPS-induced TNF- $\alpha$ and IL-6, but such anti-inflammatory activity was attenuated in the high dose group. In the clinical setting, CWE is used in combination with other herbal agents and thus different results could be produced. However, our experimental data imply that when used singularly the anti-inflammatory activity of CWE is subjected to dose ranges.

Chronic inflammatory responses found in most autoimmune diseases and metabolic diseases exhibit common characteristic processes where macrophages are initially activated and interferon (IFN)- $\gamma$-producing type$1 \mathrm{~T}$ helper cells subsequently stimulate macrophages to release more inflammatory cytokines. Together with our previous findings that CWE prevented anti-CD3stimulated $\mathrm{T}$ cells from secreting IFN- $\gamma$, our current study clearly shows that CWE is able to interfere with the chronic activation of macrophages [16].

The inhibitory effect of CWE on the signaling pathways mediated by NF-KB and MAP kinases occurred in its polyphenol-rich high MW fraction. There is increasing evidence that polyphenols exert anti-inflammatory effects. Since CWE is rich in polyphenols such as flavonoids and tannins, the anti-inflammatory effect of CWE may originate partly from polyphenolic compounds.
Although the high MW fraction accounts for only 25\% of the yield of CWE, its polyphenol content is four times more than that of CWE. The high MW fraction may contain polyphenols conjugated with polysaccharides or tannins. Procyanidins, known as condensed tannins, consist of oligomer or polymers of (epi)catechin. A higher degree of polymerized procyanidins exhibited stronger inhibition of macrophage activity [22]. Therefore, it is conceivable that the polyphenol-rich high MW fraction of CWE may contain the anti-inflammatory compounds that play a major role in suppressing LPSinduced $\mathrm{NFK}_{\mathrm{B}}$ and MAP kinase signaling pathways. Further study is required to examine whether the macromolecular polyphenols of CWE exert these antiinflammatory effects in animal models.

\section{Conclusions}

In summary, oral treatment of CWE decreased LPSinduced TNF- $\alpha$ and IL- 6 release in serum. CWE inhibited $\mathrm{I} \kappa \mathrm{B} \alpha$ degradation and MAP kinase activation in LPS-stimulated macrophages in vitro. In particular, the inhibitory activity of CWE in vitro occurred in the polyphenol-rich high molecular weight fraction.

\section{Abbreviations \\ CWE: Cinnamon water extract; LPS: Lipopolysaccharide; TNF-a: Tumor necrosis factor- a; IL: Interleukin; DMSO: Dimethyl sulfoxide; ELISA: Enzyme- linked immunosorbent assay; IKK: IKBa kinase; MAP: Mitogen-activated protein; MW: Molecular weight; PRR: Pattern recognition receptor; TLR: Toll-like receptor; IFN: Interferon.}

\section{Competing interests}

The authors have no conflict of interests.

\section{Authors' contributions}

JWH and HK have written the paper. HK and GAY performed in vitro experiments. YBK performed animal experiments. SHE assisted in the preparation of CWE. JHL assisted in the preparation of manuscript. All authors have read and approved the final manuscript.

\section{Author details}

${ }^{1}$ Graduate School of East-west Medical Science, Kyung Hee University, Yongin, Gyeonggi 446-701, South Korea. ${ }^{2}$ College of Oriental Medicine, Kyung Hee University, Seoul 130-701, South Korea. ${ }^{3}$ College of Life Science, Kyung Hee University, Yongin, Gyeonggi 446-701, South Korea.

Received: 19 July 2012 Accepted: 24 November 2012

Published: 28 November 2012

\section{References}

1. Oussalah M, Caillet S, Lacroix M: Mechanism of action of Spanish oregano, Chinese cinnamon, and savory essential oils against cell membranes and walls of Escherichia coli 0157:H7 and Listeria monocytogenes. J Food Prot 2006, 69(5):1046-1055.

2. Zhuang M, Jiang H, Suzuki Y, Li X, Xiao P, Tanaka T, Ling H, Yang B, Saitoh $H$, Zhang $L$, et al: Procyanidins and butanol extract of Cinnamomi Cortex inhibit SARS-CoV infection. Antiviral Res 2009, 82(1):73-81.

3. Kwon HK, Hwang JS, So JS, Lee CG, Sahoo A, Ryu JH, Jeon WK, Ko BS, Im CR, Lee $\mathrm{SH}$, et al: Cinnamon extract induces tumor cell death through inhibition of NFkappaB and AP1. BMC cancer 2010, 10:392-401.

4. Schoene NW, Kelly MA, Polansky MM, Anderson RA: Water-soluble polymeric polyphenols from cinnamon inhibit proliferation and alter cell cycle distribution patterns of hematologic tumor cell lines. Cancer Lett 2005, 230(1):134-140. 
5. Kim SH, Hyun SH, Choung SY: Anti-diabetic effect of cinnamon extract on blood glucose in db/db mice. J Ethnopharmacol 2006, 104(1-2):119-123.

6. Qin B, Nagasaki M, Ren M, Bajotto G, Oshida Y, Sato Y: Cinnamon extract (traditional herb) potentiates in vivo insulin-regulated glucose utilization via enhancing insulin signaling in rats. Diabetes Res Clin Pract 2003, 62(3):139-148.

7. Nathan C: Points of control in inflammation. Nature 2002, 420(6917):846-852.

8. Gaestel M, Kotlyarov A, Kracht M: Targeting innate immunity protein kinase signalling in inflammation. Nature Rev 2009, 8(6):480-499.

9. Lee HS, Kim BS, Kim MK: Suppression effect of Cinnamomum cassia bark-derived component on nitric oxide synthase. J Agric Food Chem 2002, 50(26):7700-7703.

10. Hayashi K, Imanishi N, Kashiwayama Y, Kawano A, Terasawa K, Shimada Y, Ochiai H: Inhibitory effect of cinnamaldehyde, derived from Cinnamomi cortex, on the growth of influenza A/PR/8 virus in vitro and in vivo. Antiviral Res 2007, 74(1):1-8.

11. Cabello CM, Bair WB 3rd, Lamore SD, Ley S, Bause AS, Azimian S, Wondrak GT: The cinnamon-derived Michael acceptor cinnamic aldehyde impairs melanoma cell proliferation, invasiveness, and tumor growth. Free Radic Biol Med 2009, 46(2):220-231.

12. Anderson RA, Broadhurst CL, Polansky MM, Schmidt WF, Khan A, Flanagan VP, Schoene NW, Graves DJ: Isolation and characterization of polyphenol type-A polymers from cinnamon with insulin-like biological activity. J Agric Food Chem 2004, 52(1):65-70.

13. Lu J, Zhang K, Nam S, Anderson RA, Jove R, Wen W: Novel angiogenesis inhibitory activity in cinnamon extract blocks VEGFR2 kinase and downstream signaling. Carcinogenesis 2009, 31(3):481-488.

14. Okada Y, Okada M, Sagesaka Y: Screening of dried plant seed extracts for adiponectin production activity and tumor necrosis factor-alpha inhibitory activity on 3T3-L1 adipocytes. Plant Foods Hum Nutr 2010, 65(3):225-232.

15. Cairns CB, Panacek EA, Harken AH, Banerjee A: Bench to bedside: tumor necrosis factor-alpha: from inflammation to resuscitation. Acad Emerg Med 2000, 7(8):930-941.

16. Lee BJ, Kim YJ, Cho DH, Sohn NW, Kang H: Immunomodulatory effect of water extract of cinnamon on anti-CD3-induced cytokine responses and p38, JNK, ERK1/2, and STAT4 activation. Immunopharmacol Immunotoxicol 2011, 33(4):714-722.

17. Schepetkin IA, Quinn MT: Botanical polysaccharides: macrophage immunomodulation and therapeutic potential. Int Immunopharmacol 2006, 6(3):317-333

18. Hayden MS, Ghosh S: Shared principles in NF-kappaB signaling. Cell 2008, 132(3):344-362.

19. Lee YG, Chain BM, Cho JY: Distinct role of spleen tyrosine kinase in the early phosphorylation of inhibitor of kappaB alpha via activation of the phosphoinositide-3-kinase and Akt pathways. Int J Biochem Cell Biol 2009, 41(4):811-821.

20. Kyriakis JM, Avruch J: Mammalian mitogen-activated protein kinase signal transduction pathways activated by stress and inflammation. Physiol Rev 2001, 81(2):807-869.

21. Gordon S: The macrophage: past, present and future. Eur J Immunol 2007, 37(Suppl 1):S9-S17.

22. Terra X, Valls J, Vitrac X, Merrillon JM, Arola L, Ardevol A, Blade C, Fernandez-Larrea J, Pujadas G, Salvado J, et al: Grape-seed procyanidins act as antiinflammatory agents in endotoxin-stimulated RAW 264.7 macrophages by inhibiting NFkB signaling pathway. J Agric Food Chem 2007, 55(11):4357-4365.

doi:10.1186/1472-6882-12-237

Cite this article as: Hong et al:: Anti-inflammatory activity of cinnamon water extract in vivo and in vitro LPS-induced models. BMC Complementary and Alternative Medicine 2012 12:237.

\section{Submit your next manuscript to BioMed Central and take full advantage of:}

- Convenient online submission

- Thorough peer review

- No space constraints or color figure charges

- Immediate publication on acceptance

- Inclusion in PubMed, CAS, Scopus and Google Scholar

- Research which is freely available for redistribution

Submit your manuscript at www.biomedcentral.com/submit
Ciomed Central 